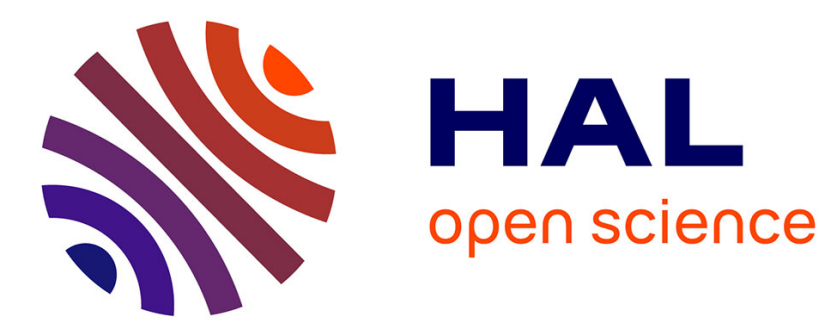

\title{
An Enhanced Spatial Reasoning Ontology for Maritime Anomaly Detection
}

Arnaud Vandecasteele, Aldo Napoli

\section{To cite this version:}

Arnaud Vandecasteele, Aldo Napoli. An Enhanced Spatial Reasoning Ontology for Maritime Anomaly Detection. 7th International Conference on System Of Systems Engineering - IEEE SOSE 2012, Jul 2012, Genoa, Italy. p. 247-252, 10.1109/SYSoSE.2012.6384120 . hal-00747507

HAL Id: hal-00747507

https://hal-mines-paristech.archives-ouvertes.fr/hal-00747507

Submitted on 6 Dec 2012

HAL is a multi-disciplinary open access archive for the deposit and dissemination of scientific research documents, whether they are published or not. The documents may come from teaching and research institutions in France or abroad, or from public or private research centers.
L'archive ouverte pluridisciplinaire HAL, est destinée au dépôt et à la diffusion de documents scientifiques de niveau recherche, publiés ou non, émanant des établissements d'enseignement et de recherche français ou étrangers, des laboratoires publics ou privés. 


\section{An Enhanced Spatial Reasoning Ontology for Maritime Anomaly Detection}

\author{
Arnaud Vandecasteele \\ MINES ParisTech \\ Centre for Crisis and Risk Research \\ Sophia Antipolis, France \\ arnaud.van_de_casteele@mines-paristech.fr
}

\author{
Aldo Napoli \\ MINES ParisTech \\ Centre for Crisis and Risk Research \\ Sophia Antipolis, France \\ aldo.napoli@mines-paristech.fr
}

\begin{abstract}
Although originally conceived as a conceptual object for modelling knowledge, current ontologies do not make it possible to manipulate spatial knowledge. However, spatial knowledge is an essential component of any modelling specification. This problem provided the motivation for the creation of an expert system driven by an ontology. The system enables experts in the maritime domain to characterise abnormal ship behaviour based on formal semantic properties. Users are able to specify and execute spatial rules that are directly integrated into the ontology and a map interface linked to the ontology displays the results of the inferences obtained.
\end{abstract}

Keywords: Spatial Ontology, Spatial Reasoning, Abnormal Maritime Behaviour

\section{Introduction}

Created in the early 1990 s as a response to the various limitations identified in the development of expert systems, ontologies appeared as a new conceptual approach to knowledge modelling. This logical object made it possible to explicitly define not only concepts capable of describing the real world but also the rules governing the structure of these descriptions [1].

Nevertheless, the modelling and interpretation of spatial knowledge is still not sufficiently exploited in ontologies. Therefore, this paper proposes an extension to the initial capabilities of the Semantic Web Rule Language (SWRL) through the integration of spatial reasoning procedures. These procedures are incorporated into a Geographic Information System (GIS) driven by an ontology [2]. The approach is validated by the implementation of a platform capable of detecting abnormal ship behaviour.

We first describe the domain of ontologies and that of spatial ontologies (Section 2). Next, we discuss trajectory modelling and how this can be semantically enriched (Section 3). Finally, we present the prototype developed for the automatic detection of abnormal ship behaviour based on a spatial ontology (Section 4).

\section{The contribution of ontologies to knowledge modelling}

Although originally associated with the domain of philosophy, in this paper we examine the concept of ontology from the perspective of Artificial Intelligence (AI). Designed as a response to the problems posed by knowledge integration, ontologies appeared as a key paradigm in solving the problem of semantic heterogeneity and ensuring interoperability, as much between systems as between individuals [3].

This dual view of ontologies, which represent both a compendium of knowledge and an information object, is found in the definition given by Studer [4] for whom, "An ontology is a formal and explicit specification of a shared conceptualization." Formal indicates that the conceptualisation and representation of the domain should be standardised and usable by an information system. Explicit specifies that the concepts used as constraints are defined declaratively. Conceptualization emphasises the fact that an ontology is only a partial abstraction of the real world. Finally, the notion of sharing implies that ontologies facilitate consensual knowledge.

The domain of ontologies therefore provided the necessary structures for knowledge modelling. Consequently, various ontology languages with different semantic capabilities were created.

\subsection{Ontology languages}

An ontology language makes it possible to express an interpretation of the world based on formal semantics and a precise syntactic structure. Since the 1990s there has been a proliferation of so-called traditional languages (Ontolingua, Cycl, Loom, etc.) coming from the field of AI [5], which it became necessary to standardise. Consequently, under the leadership of the OntoWeb group of the World Wide Web Consortium (W3C), 
standardised languages have been defined; examples include the Resource Description Framework (RDF) and the Web Ontology Language (OWL) [6]. OWL was implemented in the platform we have developed and we will briefly describe it here.

OWL has been the defined W3C standard for ontology creation since 2004. Based on the DAML+OIL language, OWL is founded on the basic primitives defined in RDF schemas. Nevertheless, far from being a simple extension of RDF, it provides all the semantics needed to describe knowledge such as mechanisms for the comparison of classes (concepts of equivalence, symmetry, etc.). Rather than defining a modelling language that was complex and difficult to use, the W3C decided to provide three increasingly expressive OWL sublanguages: OWL Lite, OWL DL and OWL Full. Each of these sublanguages is itself an extension of its predecessor [6]. In 2009, a new version of OWL (OWL 2) was proposed by the W3C, which aimed to be both an extension and revision of OWL. The motivation for the development of this new version came from the limited expressiveness of OWL, an overly complex syntax and the inability to annotate axioms [7].

Another limitation of OWL related to the absence of syntactic structures for rule creation. However, it is these structures that enable reasoning and the deduction of new facts from information contained in a knowledge base. Consequently, Horrocks [8] proposed the creation of the Semantic Web Rule Language (SWRL) that combines OWL DL and RuleML. Designed to support reasoning based on descriptive logic and Horn rules, the structure of a SWRL rule takes the form antecedent $\rightarrow$ consequent which is read as, "If the antecedent is true, then the consequent is also true". Unlike OWL, SWRL only allows the addition of relationships and existing properties if they meet the rule. In addition to the OWL predicates, SWRL has supplementary 'built-in' functions. These functions extend the initial OWL capabilities; in particular they enable string comparisons and calculations. We have adopted this idea of built-in functions in order to integrate spatial capabilities into SWRL rules.

\subsection{The spatial dimension of ontologies}

The term 'geographic ontology' brings together two disciplines and worlds that employ different concepts; that of ontologies (which we have already discussed) and that of geography in the broad sense. However, as Agarwal [9] points out, the various existing studies that aim to bring together Geographic Information Systems and ontologies are essentially focussed on the strengths of each domain and do not form a true common discipline. For example (although the project is under consideration by the $\mathrm{W} 3 \mathrm{C}$ ) there is currently no standard for the representation of spatial data in ontologies or for spatial reasoning procedures in inference engines. As a result, various initiatives (e.g. SWEET ${ }^{1}$, Ordnance Survey Ontologies ${ }^{2}, \mathrm{SWING}^{3}$, etc.) have attempted to define the necessary characteristics that must be implemented in spatial ontologies. However, these projects vary widely in terms of intended usage, the formalisation of representation and the rigor of the philosophical assumptions employed [10]. Consequently, the Geospatial Incubator Group (GeoXG), a W3C Working Group, is currently working on defining future directions for the integration of the spatial dimension into ontologies.

One of the first measures taken by the group was focused on the adoption of GeoRSS as a recommendation for the description of the geospatial properties of Web resources [11]. The result of the work of the GeoXG group, GeoRSS is inspired by GML while at the same time simplifying it in order to be as generic as possible. While the use of GeoRSS in the domain of ontologies offers the advantage of a simplified spatial representation, it nevertheless tends to suffer from an overly limited semantic. Moreover, beyond the formalisation of spatial entities, very little work has been carried out concerning the implementation of spatial reasoning procedures in ontologies [12].

\section{Semantic modelling of trajectories}

Although the study of moving objects is found in many domains, the principal contributions have come from the database community. These contributions particularly relate to the definition of new data types and operator-specific queries that can represent and query moving objects using a Database Management System (DBMS) [13]. The formalisation and conceptualisation of trajectories in an information system is a necessary but insufficient step in their understanding, use and analysis. Various approaches have been proposed in order to address these limitations including, notably, better integration of the semantics associated with trajectories [14]. In the context of this article, this is also the approach we have taken.

\subsection{From trajectories to semantic trajectories}

As defined by Spaccapietra [15], the concept of the semantic trajectory makes it possible to extract from raw data, trajectories whose components have been enriched by different types of information.

1 Semantic Web for Earth and Environmental Terminology (http://sweet.jpl.nasa.gov/ontology/)

${ }^{2}$ http://www.ordnancesurvey.co.uk/oswebsite/ontology/

${ }^{3}$ Semantic Web Services Interoperability for Geospatial Decision Making (http://swing.brgm.fr/repository/ontologies) 
A semantic trajectory is therefore defined as the movement of an entity in a geographic area, in a given time period. Therefore, it is marked by periods of movement and stops. The concept of the stop applied to a moving object means that trajectories can be defined, both temporally and spatially. The move is the time period between two stops during which an object is moving in space. It is thus defined by a sequence of temporally ordered positions. Each of these elements can be represented in ontological form [16] (Figure 1).

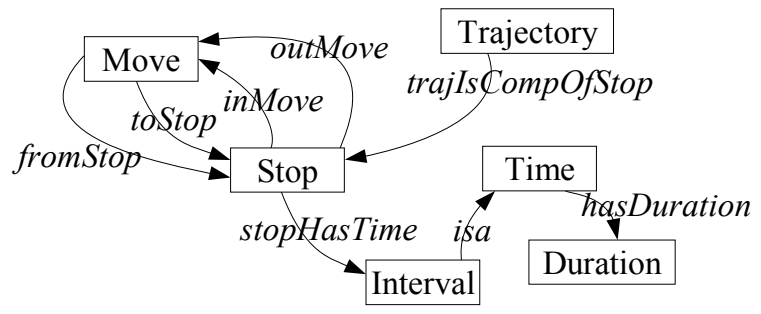

Figure 1. Ontological representation of the concept of semantic trajectories (based on [16])

As defined above, Figure 1 shows the two elements Stop and Move which delineate a trajectory both spatially and temporally. Therefore, in the approach taken by Baglioni et al. [16], the conceptualisation of a trajectory is represented by a stop sequence associated with a movement. This connection is made using one of four relationships: fromStop, toStop, inMove, outMove. In addition, each Stop takes place in a specific time dimension which is defined by the relation stopHasTime.

\subsection{The semantic enrichment of trajectories}

Using this ontological model, supplementary information can be added to the trajectory components. This semantic enrichment process generates, from the raw data, trajectories whose content has been enriched by the addition of related information - for example the geographical zone or domain of activity (Figure 2). Moreover, the user must be able to directly manipulate these semantic trajectories using an expressive language that can be understood by humans [16].

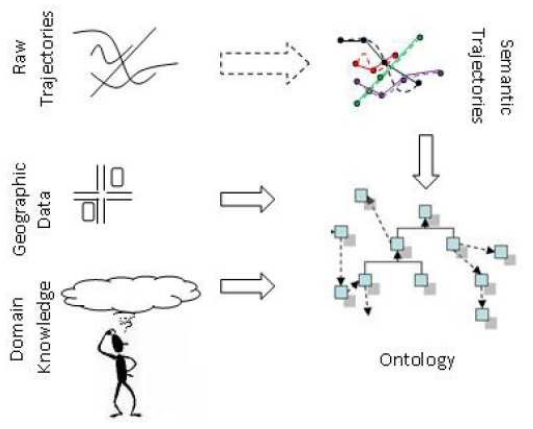

Figure 2. The process for the semantic enrichment of trajectories (based on [16])
Based on a similar logic of semantic enrichment, Yan [17] suggests a comprehensive architecture for the creation, management and analysis of trajectories. The architecture relies on a modular infrastructure which consists of three principal ontologies: a geometric trajectory ontology, a geographic ontology and a domain application ontology. These three ontologies are then combined to provide the semantic infrastructure necessary to describe the trajectory. We have adopted this approach for the creation of the ontology for the detection of abnormal ship behaviour.

\section{The detection of abnormal ship behaviour using trajectory analysis}

\subsection{Prototype architecture}

The principal idea of the prototype is to enable users to specify abnormal ship behaviour in relation to their trajectory. The prototype described in this paper is based on an architecture similar to that proposed by Yan [17]. The analysis of abnormal ship behaviour consists of four stages (Figure 3). First, databases are updated as new information arrives (step 1). The new information is added to the ontology (step 2). Then, the inference engine characterises the behaviour of each vessel taking into account the new information and the rules previously defined (step 3). Finally, a mapping module displays the result of the inference (step 4). Steps 2, 3 and 4 form the heart of our system and they are described in greater detail in the following paragraphs.

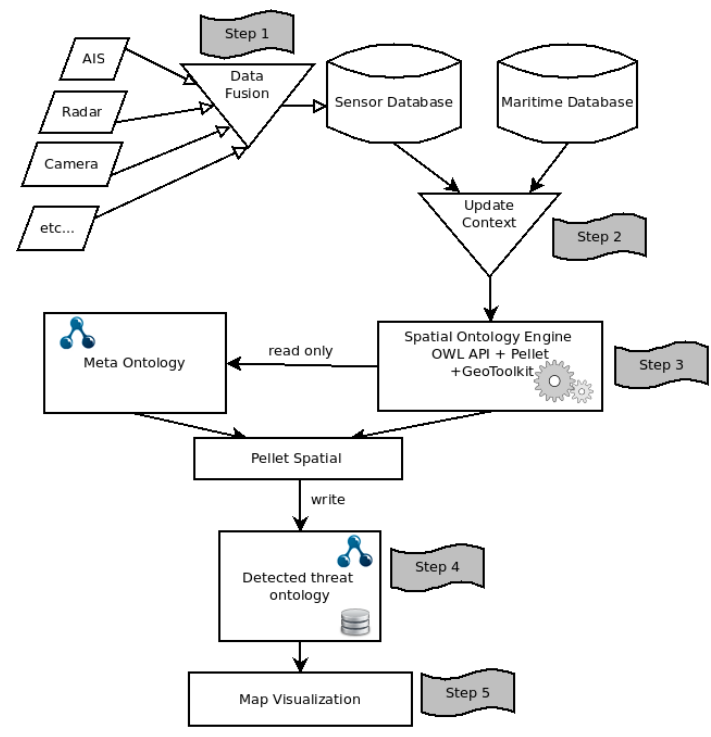

Figure 3. System architecture for the detection of anomalous ship behaviour

The first step is to update the ontology based on new information supplied by sensors. This step integrates 
dynamic and static data in the knowledge base. This is achieved through the OWL-API ${ }^{4}$ Java library that provides a high-level programming interface for the creation and manipulation of ontologies.

Next, in order to be exploited, the ontology must be integrated into a reasoning engine. The purpose of this engine is to infer new facts from existing data. In this case, it will provide an evaluation of the situation taking into account the positions and characteristics of ships. From the various reasoners currently available, we decided to use Pellet ${ }^{5}$. This application has very good SWRL language support, it is compatible with the OWL 2 language and it integrates seamlessly with the JENA and OWL-API libraries. Above all, it is to our knowledge, the only application that enables the addition of new built-in functions in order to create custom SWRL rules [18]. Using class instances found in the knowledge base, the reasoner applies the various SWRL rules that have been defined. Ship behaviour can then be determined according to the outcome of the application of these rules.

Finally, once the ontology has been updated, the result of the inference can be displayed on a map interface. The map interface is directly linked to the ontology and provides a visualisation of the analyses using a customised graphical semiology. For example, the criticality level of a ship is indicated by graduated colours. Moreover, other information such as the vessel properties, its spatial characteristics and specified alerts can be displayed.

\subsection{Experimentation and definition of detection rules}

The experimental data consisted of records of ship positions for vessels equipped with an Automatic Identification System (AIS) in the Mediterranean Sea. It totalled more than 360,000 coordinates recorded during one week. Equipped with this dataset, the goal was then to detect abnormal ship behaviour. To achieve this, a set of rules was defined using both knowledge from domain experts and data contained in the scientific literature [1921].

In the ontology, two types of rules can be distinguished: non-spatial and spatial. Non-spatial rules directly exploit SWRL reasoning capabilities. For example, it might be necessary to detect a vessel moving at an excessive speed for its type. The request, translated into SWRL, is shown below and reads as follows: "If the ship's speed (?vesselType) is greater than (greaterThan) the maximum speed for this type of vessel

\footnotetext{
${ }^{4}$ http://owlapi.sourceforge.net/

${ }^{5} \mathrm{http}: / /$ clarkparsia.com/pellet/
}

(?speedTypeVessel) then trigger an alert (Alert_Speed_HighSpeed)"

Vessel (?vessel),

Vessel_hasVesselType (?vessel, ?vesselType),

Vessel_hasSpeed (?vessel, ?speedVessel),

Vessel_hasSpeed (?vesselType, ?speedTypeVessel), greaterThan (?speedTypeVessel, ?speedVessel) $\rightarrow$ Vessel_hasAlert (?vessel, Alert_Speed_HighSpeed)

As we noted earlier, the analysis of abnormal ship behaviour must also take into account spatial dimensions. This element is one of the major contributions of our research. To achieve this, we extended the traditional functions of SWRL, creating custom built-in spatial functions (intersects, touches, etc.). The integration of these built-in functions was achieved using the expansion capabilities of the Pellet reasoning engine. The following is a concrete example:

Vessel(?vessel),

Vessel_hasPosition(?vessel, ?position),

Analysis_DataPath(Alert_Area_Restricted, ?geoData),

intersects(?geoData, ?position) $\rightarrow$

Vessel_hasAlert(?vessel, Alert_Area_Restricted)

In this example, we perform an intersection (intersects) between the position of the ship (?position) and geographic data specified in the alert class (?geoData). Then, if the result is positive, we add an object property between the detected ship and the specified alert (Alert_Area_Restricted). Obviously, various spatial functions and other datasets are available. To model a different spatial function, it is simply a case of changing the keyword in the SWRL rule and for the data, specifying which data to use from the data property DP_Analysis_DataPath.

\subsection{Examples of abnormal behaviour}

To illustrate the operation of the ontology-driven GIS we chose the following two cases (Figure 4). First, we take the simple example of an intersection between the position of a ship and a restricted zone. The second case is more complex as it refers not only to the position, but also the trajectory of two ships.

In the first example, which relates to the restricted zone (Figure $4 \mathrm{a}$ ) the inference engine has automatically detected an offence based on the ship's position and the geographic data specified in the ontology. The offending vessel is then reported as suspect. In addition, the alert criticality index automatically assigns a danger level to the ship.

In the second case (Figure 4b) there are two vessels on a parallel course. In the maritime domain, this could indicate a potential collision or a pair of fishing vessels. As before, the inference engine analyses the trajectories of the vessels and provides a geometric similarity index based on the mathematical formula of Haversine [22]. 
Depending on the outcome of this index, an alert is automatically added to the vessels involved. It should also be noted that (in addition to being on a parallel trajectory) the two ships are in a restricted zone. This particular gradation of dangerousness is highlighted by the orange colour that surrounds each of the two ships.
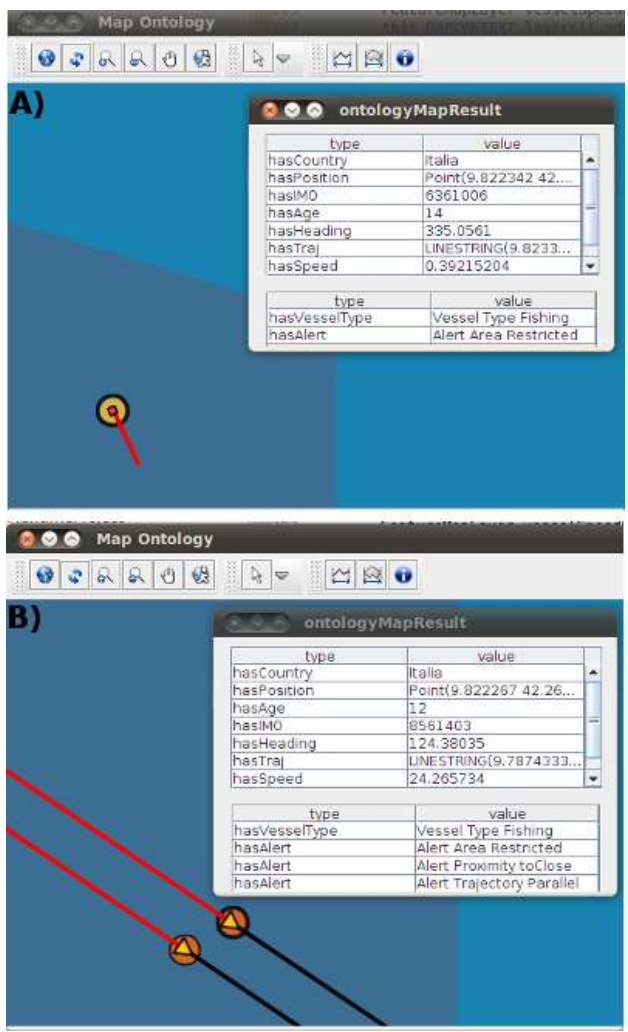

Figure 4. Examples of abnormal ship behaviour. A) Presence of a ship in a restricted area. B) Detection of parallel trajectories

\section{Conclusion}

This paper proposes the integration of the spatial dimension into an ontology in order to enable experts in the maritime domain to specify rules governing abnormal ship behaviour. To achieve this, we enriched the semantics of the SWRL language, which made it possible to define spatial functions. To validate our research hypothesis, the proposed solution was integrated into a GIS driven by an ontology. The prototype system aims to analyse ship positions and characterise their behaviour according to rules defined by experts.

The prototype described here is functional and can simultaneously analyse the behaviour of many thousands of ships. Nevertheless, the results of experiments carried out so far suggest that a useful improvement would be to facilitate the step of creating detection rules, which must currently be written using SWRL syntax. The creation of these rules can be a significant constraint for domain experts responsible for creating the model. Therefore, it may be useful to provide a graphical interface for rule creation along the lines of the Snoggle interface (Snoggle is a graphical, SWRL-based ontology mapper). The expert would then only have to draw abnormal behaviour, which would be translated into the SWRL language before being integrated into the ontology.

As we have demonstrated, the integration of the spatial dimension is an essential element in the structuring of knowledge. Nevertheless, it cannot yet be fully implemented in ontologies due to the lack of appropriate structures. Ultimately, it appears that it will be necessary to make use of spatial data types such as those currently found in geographic databases.

\section{References}

[1] F. Gandon, "Graphes RDF et leur Manipulation pour la Gestion de Connaissances (RDF Graphs and their Manipulation for Knowledge Management)", Habilitation à Diriger des Recherches, INRIA, 2008.

[2] F. T. Fonseca and M. J. Egenhofer, "Ontologydriven geographic information systems", in Proceedings of the 7th ACM International Symposium on Advances in Geographic Information Systems, New York, NY, USA, pp. 14-19, 1999.

[3] T. Mondary, “Construction d'ontologies à partir de textes. L'apport de l'analyse de concepts formels (The Construction of Ontologies from Texts. The Contribution of Formal Methods)", Phd, 27 May 2011.

[4] R. Studer, V. R. Benjamins, and D. Fensel, "Knowledge engineering: Principles and methods", Data \& Knowledge Engineering, Vol. 25, No. 1-2, pp. 161197, March 1998.

[5] V. Nguyen, "Ontologies and Information Systems: A Literature Survey", Defence Science and Technology Organisation, Technical Note DSTO-TN1002, June 2011.

[6] S. Bechhofer, F. van Harmelen, J. Hendler, I. Horrocks, D. L. McGuinness, P. F. Patel-Schneider, and L. A. Stein, "OWL Web Ontology Language Reference". W3C, 10 February 2004.

[7] B. C. Grau, I. Horrocks, B. Motik, B. Parsia, P. Patel-Schneider, and U. Sattler, "OWL 2: The next step for OWL", Web Semantics: Science, Services and Agents on the World Wide Web, Vol. 6, No. 4, pp. 309322, November 2008. 
[8] I. Horrocks, P. Patel-Schneider, H. Boley, S. Tabet, B. Grosof, and M. Dean, "SWRL: A Semantic Web Rule Language Combining OWL and RuleML". W3C Report, 2004.

[9] P. Agarwal, "Ontological considerations in GIScience", International Journal of Geographical Information Science, Vol. 19, No. 5, pp. 501-536, 2005.

[10] J. Ressler, M. Dean, and D. Kolas, "Geospatial Ontology Trade Study", National GeospatialIntelligence Agency, Report No. HM1582-05-C-0014, 2007.

[11] J. Lieberman, R. Singh, and C. Goad, "W3C Geospatial Ontologies - W3C Incubator Group", W3C Incubator Group, Report, 2007.

[12] A. Karmacharya, C. Cruz, F. Boochs, and F. Marzani, "Use of geospatial analyses for semantic reasoning", in Proceedings of the 14th International Conference on Knowledge-based and Intelligent Information and Engineering Systems: Part I, Berlin, Heidelberg, pp. 576-586, 2010.

[13] V. Mallé-Noyon, "Modèle de vue relative et spatio-temporelle de trajectoires géographiques d'objets mobiles: application au contexte maritime (Relative and spatio-temporal model of the geographical trajectories of moving objects: application to the maritime context)", Ecole Nationale Supérieure d'Arts et Métiers, Phd, 2007.

[14] J. Malki, W. Mefteh, and A. Bouju, "Une approche ontologique pour la modélisation et le raisonnement sur les trajectoires. Prise en compte des règles métiers, spatiales et temporelles (An ontological approach for trajectory modelling and reasoning)", in JFO 2009 3ème édition des journées Francofones sur les Ontologies, France, pp. 157-168, 2009.

[15] S. Spaccapietra, C. Parent, M. L. Damiani, J. A. de Macedo, F. Porto, and C. Vangenot, "A conceptual view on trajectories", Data \& Knowledge Engineering, Vol. 65, No. 1, pp. 126-146, April 2008.

[16] M. Baglioni, J. Macedo, C. Renso, and M. Wachowicz, "An Ontology-Based Approach for the Semantic Modelling and Reasoning on Trajectories", in Advances in Conceptual Modeling - Challenges and Opportunities, Vol. 5232, Springer Berlin/Heidelberg, pp. 344-353, 2008.

[17] Z. Yan, "Semantic Trajectories: Computing and Understanding Mobility Data", Phd, Ecole Polytechnique Fédérale de Lausanne, 2011.
[18] E. Sirin, B. Parsia, B. C. Grau, A. Kalyanpur, and Y. Katz, "Pellet: A practical OWL-DL reasoned", Web Semantics, Vol. 5, No. 2, pp. 51-53, June 2007.

[19] J. Van Laere and M. Nilsson, "Evaluation of a workshop to capture knowledge from subject matter experts in maritime surveillance", in Proceedings of the 12th International Conference on Information Fusion, 2009. FUSION '09, pp. 171-178, 2009.

[20] B. Idiri and A. Napoli, "Découverte de règles d'association pour l'aide à la prévision des accidents maritimes (The Discovery of Association Rules to Help in the Prediction of Maritime Accidents)", paper presented at the Extraction et Gestion des Connaissances (EGC), Bordeaux, 2012.

[21] X. Chaze, A. Bouejla, and F. Guarnieri, "The contribution of bayesian networks to manage risks of maritime piracy against oil offshore fields", paper presented at the 17th International Conference on Database Systems for Advanced Applications (DASFAA), South Korea, 15-19 April 2012.

[22] T. Devogele, "Système d'information géographique temporelle maritime; Des distances linéaires à l'analyse temps réel des trajectoires (Maritime Temporal Geographic Information Systems; Linear Realtime Analysis of Trajectories) ", Habilitation à Diriger des Recherches, Université de Bretagne Occidentale, 2009. 